\title{
E- and B-mode mixing from incomplete knowledge of the shear correlation (Research Note)
}

\author{
M. Kilbinger, P. Schneider, and T. Eifler
}

\author{
Argelander-Institut für Astronomie ${ }^{\star}$, Universität Bonn, Auf dem Hügel 71, 53121 Bonn, Germany \\ e-mail: kilbinge@astro.uni-bonn.de
}

Received 25 April 2005 / Accepted 20 June 2006

\begin{abstract}
Aims. We quantify the mixing of the measured cosmic-shear E- and B-modes caused by the lack of shear-correlation measurements on small and large scales, arising from a lack of close projected galaxy pairs and the finite field size, respectively.

Methods. We calculate the aperture-mass statistics $\left\langle M_{\mathrm{ap}, \perp}^{2}\right\rangle$ and the E-/B-mode shear-correlation functions $\xi_{\mathrm{E}, \mathrm{B} \pm}$ where small- and large-scale cutoffs are taken into account. We assess the deviation of the obtained E-mode to the true E-mode and the introduction of a spurious B-mode.

Results. The measured aperture-mass dispersion is underestimated by more than $10 \%$ on scales smaller than 12 times the lower cutoff. For a precise measurement of the E- and B-modes at the percent level using a combination of $\xi_{\mathrm{E}, \mathrm{B}+}$ and $\xi_{\mathrm{E}, \mathrm{B}-}$, a field as large as 7 (2.4) degrees is necessary for ground-based (space-based) observations.
\end{abstract}

Key words. gravitational lensing - large-scale structure of Universe - cosmological parameters

\section{Introduction}

The observation of the correlation between the shapes and orientation of high-redshift galaxies has become a well-established method of studying the dark matter distribution on very large scales. Using theoretical predictions for the power spectrum of the projected cosmic density field, cosmological parameters can be obtained from the measurement of cosmic shear, most notably the matter density $\Omega_{\mathrm{m}}$ and the amplitude of density fluctuations $\sigma_{8}$. Very recent results include ground-based (Hoekstra et al. 2006; Massey et al. 2005; Semboloni et al. 2006; Jarvis et al. 2006) and space-based (Rhodes et al. 2004; Heymans et al. 2005) observations.

Presently, cosmic-shear surveys are still limited by systematic errors arising from the imperfect shape measurement of faint galaxies and a deficient PSF correction. Extensive studies and comparisons between different data analysis methods are being made (Heymans et al. 2006a) to find sources of any contamination of the cosmological signal. A common means to check for systematics in the data is the decomposition of the measured shear field (or power spectrum) into the gradient- and curl-parts (E- and B-modes), see Crittenden et al. (2002, hereafter C02), and Schneider et al. (2002, hereafter S02). Since the image distortions caused by gravitational lensing are (to first order) curl-free, the presence of a B-mode is a distinct imprint of residuals not completely removed from the measurement. A B-mode of cosmological origin can be caused by the intrinsic correlation of galaxy orientations (e.g. Crittenden et al. 2001; Jing 2002).

^ Founded by merging of the Sternwarte, Radioastronomisches Institut, and Institut für Astrophysik und Extraterrestrische Forschung der Universität Bonn.
However, this effect is thought to be very small for reasonably deep surveys (e.g. Hirata et al. 2004), and it can be removed from the shear signal using photometric redshifts or by modeling the intrinsic alignment signal that is distinct from the shear signal (Heymans \& Heavens 2003; King \& Schneider 2002, 2003; King 2005). A further non-negligible source of confusion could be the recently discovered intrinsic shape-shear correlation (Hirata \& Seljak 2004). This contamination also creates a B-mode (Heymans et al. 2006b), and it is not yet clear how to correct for this effect.

As we present in this paper, up to now the E- and B-mode decomposition cannot be performed directly from the shear correlation as measured from the data, but it involves an integral over the shear-correlation function $\xi_{ \pm}$to either arbitrary small or infinitely large angular separations. However, the scales on which $\xi_{ \pm}$can be measured are limited. On arc-second scales, the blending of closely projected galaxy pairs prohibits a reliable determination of the shape of those galaxies. On large angular scales, the measurement is restricted by the finite field-of-view. These limits cause a mixing of the E- and B-modes with the currently-used estimators, preventing their clear-cut separation. Although this is a well-known fact, to our knowledge this mixing has not or only inadequately been taken into account to date.

In this paper, we quantify the mixing of the E- and B-mode due to the small- and large-scale limits of the shear-correlation measurements. In Sect. 2, the aperture-mass dispersion and in Sect. 3, the E-/B shear-correlation functions are considered as measures of the E- and B-mode, respectively. Section 4 briefly discusses the dependence on cosmology. In Sect. 5, we give a summary and offer ways of clearly determining the E- and the B-modes. 


\section{Aperture-mass dispersion}

The aperture-mass statistics (Kaiser et al. 1994; Schneider 1996) is a smoothed function of the convergence field,

$M_{\mathrm{ap}}(\theta)=\int \mathrm{d}^{2} \vartheta U_{\theta}(|\boldsymbol{\vartheta}|) \kappa(\boldsymbol{\vartheta})$,

where $U_{\theta}$ is a compensated filter function, i.e. $\int \mathrm{d} \vartheta \vartheta U_{\theta}(\vartheta)=$ 0 . Equation (1) can be written as an integral over the tangential shear $\gamma_{\mathrm{t}}$, weighted with the function $Q_{\theta}(\vartheta)=$ $2 / \vartheta^{2} \int_{0}^{\vartheta} \mathrm{d} \vartheta^{\prime} \vartheta^{\prime} U_{\theta}\left(\vartheta^{\prime}\right)-U_{\theta}(\vartheta)$. Moreover, $M_{\perp}$ is defined as the weighted integral over the cross-component of shear $\gamma_{\times}$,

$M_{\mathrm{ap}, \perp}(\theta)=\int \mathrm{d}^{2} \vartheta Q_{\theta}(|\boldsymbol{\vartheta}|) \gamma_{\mathrm{t}, \times}(\boldsymbol{\vartheta})$.

The dispersion of (1) (Schneider et al. 1998) can be written in terms of the convergence power spectrum $P_{K}$,

$\left\langle M_{\mathrm{ap}}^{2}\right\rangle(\theta)=\frac{1}{2 \pi} \int \mathrm{d} \ell \ell P_{\kappa}(\ell) \hat{U}^{2}(\theta \ell)$,

where $\hat{U}(\eta)$ is the Fourier transform of the filter function $u(\vartheta / \theta)=\theta^{2} U_{\theta}(\vartheta)$. The dispersion of $M_{\perp}$ is non-zero only if a curl-part or B-mode is present in the shear field. This is not the case for a purely gravitational shear signal (to first order). Therefore, the measurement of $\left\langle M_{\perp}^{2}\right\rangle$ is a test for systematic errors and/or intrinsic galaxy alignment.

Two sets of filter functions have been extensively used for cosmic-shear measurements and studies. One set are polynomial functions with finite support (Schneider et al. 1998),

$U_{\theta}(\vartheta)=\frac{9}{\pi \theta^{2}}\left(1-\frac{\vartheta^{2}}{\theta^{2}}\right)\left(\frac{1}{3}-\frac{\vartheta^{2}}{\theta^{2}}\right) \mathrm{H}\left(1-\frac{\vartheta}{\theta}\right) ; \hat{U}(\eta)=\frac{24 \mathrm{~J}_{4}(\eta)}{\eta^{2}} ;$

$Q_{\theta}(\vartheta)=\frac{6}{\pi \theta^{2}} \frac{\vartheta^{2}}{\theta^{2}}\left(1-\frac{\vartheta^{2}}{\theta^{2}}\right) \mathrm{H}\left(1-\frac{\vartheta}{\theta}\right)$;

where $\mathrm{H}$ is the Heaviside step function. The other set is related to Gaussians (C02),

$$
\begin{aligned}
& U_{\theta}(\vartheta)=\frac{1}{2 \pi \theta^{2}}\left(1-\frac{\vartheta^{2}}{2 \theta^{2}}\right) \mathrm{e}^{-\vartheta^{2} / 2 \theta^{2}} ; \quad \hat{U}(\eta)=\frac{\eta^{2}}{2} \mathrm{e}^{-\eta^{2} / 2} ; \\
& Q_{\theta}(\vartheta)=\frac{1}{4 \pi \theta^{2}} \frac{\vartheta^{2}}{\theta^{2}} \mathrm{e}^{-\vartheta^{2} / 2 \theta^{2}} .
\end{aligned}
$$

The aperture-mass dispersion can be obtained by integrating over the two-point correlation function $(2 \mathrm{PCF})$ of shear, $\xi_{ \pm}(\mathrm{C} 02$, S02),

$$
\left\langle M_{\mathrm{ap}, \perp}^{2}(\theta)\right\rangle=\frac{1}{2} \int_{0}^{\infty} \frac{\mathrm{d} \vartheta \vartheta}{\theta^{2}}\left[\xi_{+}(\vartheta) T_{+}\left(\frac{\vartheta}{\theta}\right) \pm \xi_{-}(\vartheta) T_{-}\left(\frac{\vartheta}{\theta}\right)\right] .
$$

The 2PCF are defined in terms of the tangential and crosscomponent of shear, $\gamma_{\mathrm{t}}$ and $\gamma_{\times}$, respectively, where these two components are measured with respect to the direction connecting two points. The $2 \mathrm{PCF}$ can be written as functions of the power spectrum as follows,

$\xi_{ \pm}(\vartheta)=\left\langle\gamma_{\mathrm{t}} \gamma_{\mathrm{t}}\right\rangle(\vartheta) \pm\left\langle\gamma_{\times} \gamma_{\times}\right\rangle(\vartheta)=\int_{0}^{\infty} \mathrm{d} \ell \ell P_{\kappa}(\ell) \mathrm{J}_{0,4}(\vartheta \ell)$.

The functions $T_{ \pm}$in (6) are given by

$T_{ \pm}(x)=\frac{1}{2 \pi} \int_{0}^{\infty} \mathrm{d} t t \mathrm{~J}_{0,4}(x t) \hat{U}^{2}(t)$ closed-formula expressions in the case of the above defined filter functions can be found in S02 and C02. Expression (6) is very useful since direct determination of the aperture-mass dispersion from data is very inefficient due to boundary effects and masked regions in the images. The $2 \mathrm{PCF}$, on the other hand, can be obtained easily from any survey topology. Note that, in the case of the polynomial filter, the integral in (6) only extends to $2 \theta$, since then $T_{ \pm}(x)=0$ for $x>2$.

Projected close galaxy pairs in the data have to be excluded from the shear measurements. The shape of close pairs cannot be estimated reliably because of the blending of the individual galaxy images. That means that the $2 \mathrm{PCF}$ cannot be measured for smaller angular separations than some cutoff separation $\theta_{\min }$. For space-based observations, this cutoff may be as small as one arc second. Ground-based observations usually are limited by $\theta_{\text {min }} \sim 5$ arcsec.

This inevitable cutoff at small angular scales leads to a biased estimate of the aperture-mass statistics. We introduce the quantity $\left\langle M_{\mathrm{ap}, \perp}^{2}\left(\theta, \theta_{\mathrm{min}}\right)\right\rangle$, which is defined as in (6), but with the lower integration limit replaced by $\theta_{\min }$. Even in the absence of a B-mode in the convergence field, a $\theta_{\min }>0$ will create a spurious B-mode signal. For small angular scales $\vartheta$, the first term in square brackets in (6) is approximately a positive constant, since both $\xi_{+}$and $T_{+}$are integrals over a positive function multiplied by $\mathrm{J}_{0}$. The second term tends to zero because of $\mathrm{J}_{4}$ in both $\xi_{\text {- }}$ and $T_{-}$. This will result in a negative B-mode, $\left\langle M_{\perp}^{2}\left(\theta, \theta_{\min }\right)\right\rangle\langle 0$, and an underestimation of the E-mode, $\left\langle M_{\text {ap }}^{2}\left(\theta, \theta_{\min }\right)\right\rangle<\left\langle M_{\text {ap }}^{2}(\theta)\right\rangle$. In fact, since the $T_{-}$-term is small for small scales, the approximate relation $\left\langle M_{\mathrm{ap}}^{2}\left(\theta, \theta_{\min }\right)\right\rangle-\left\langle M_{\perp}^{2}\left(\theta, \theta_{\min }\right)\right\rangle \approx\left\langle M_{\mathrm{ap}}^{2}(\theta)\right\rangle$ holds for $\theta_{\min } / \theta \ll 1$.

Note that the same effects would arise if $\left\langle M_{\mathrm{ap}, \perp}^{2}\right\rangle$ were obtained by putting apertures on the data field. In this case, the estimator of the aperture-mass dispersion involves summation over all galaxy pairs in an aperture. Due to the cutoff, this sum would lack close pairs and the E-mode would be underestimated.

Figures 1 and 2 show the influence of the cutoff $\theta_{\min }$ due to the absence of close pairs on the E- and B-modes; see Sect. 4 for the dependence on the cosmological model and source redshift distribution. In Fig. 3 we quantify the suppression of the true E-mode. Only for aperture radii $\theta$ larger than the threshold $\theta_{0}$ is the deviation smaller than indicated by the curves. For the polynomial filter, one roughly finds $\theta_{0}=12 \theta_{\min }$ for $10 \%$ accuracy. For example, for ground-based observations with $\theta_{\min }=5 \operatorname{arcsec}$ one gets deviations of more than $10 \%$ for $\theta \lesssim 1^{\prime}$. If a $1 \%$ precision is aspired scales smaller than 3:7 have to be discarded.

The Gaussian filter is less affected by the cutoff than the polynomial one, since it is broader and samples the $2 \mathrm{PCF}$ on larger angular scales for a given aperture radius. However, this advantage here turns into a disadvantage on large angular scales. There, the E- and B-modes cannot be determined reliably due to the field boundary and the infinite support of the Gaussian filter functions.

The definition of $T_{+}(8)$ shows that the mixing of the E- and B-modes due to a small-scale cutoff is inevitable. Regardless of the choice of the filter function, $U_{\theta}, T_{+}(x)$ tends to a positive value for $x \rightarrow 0$. The more rapidly $T_{+}$falls off, the smaller the bias and thus the shallower $\hat{U}$ and the broader $U_{\theta}$ is.

\section{E- and B-mode shear-correlation function}

The E- and B-mode correlation functions $\xi_{\mathrm{E}, \mathrm{B}+}$ and $\xi_{\mathrm{E}, \mathrm{B}-}$ were defined in S02 and C02. They can be obtained from the $2 \mathrm{PCF}$ in 

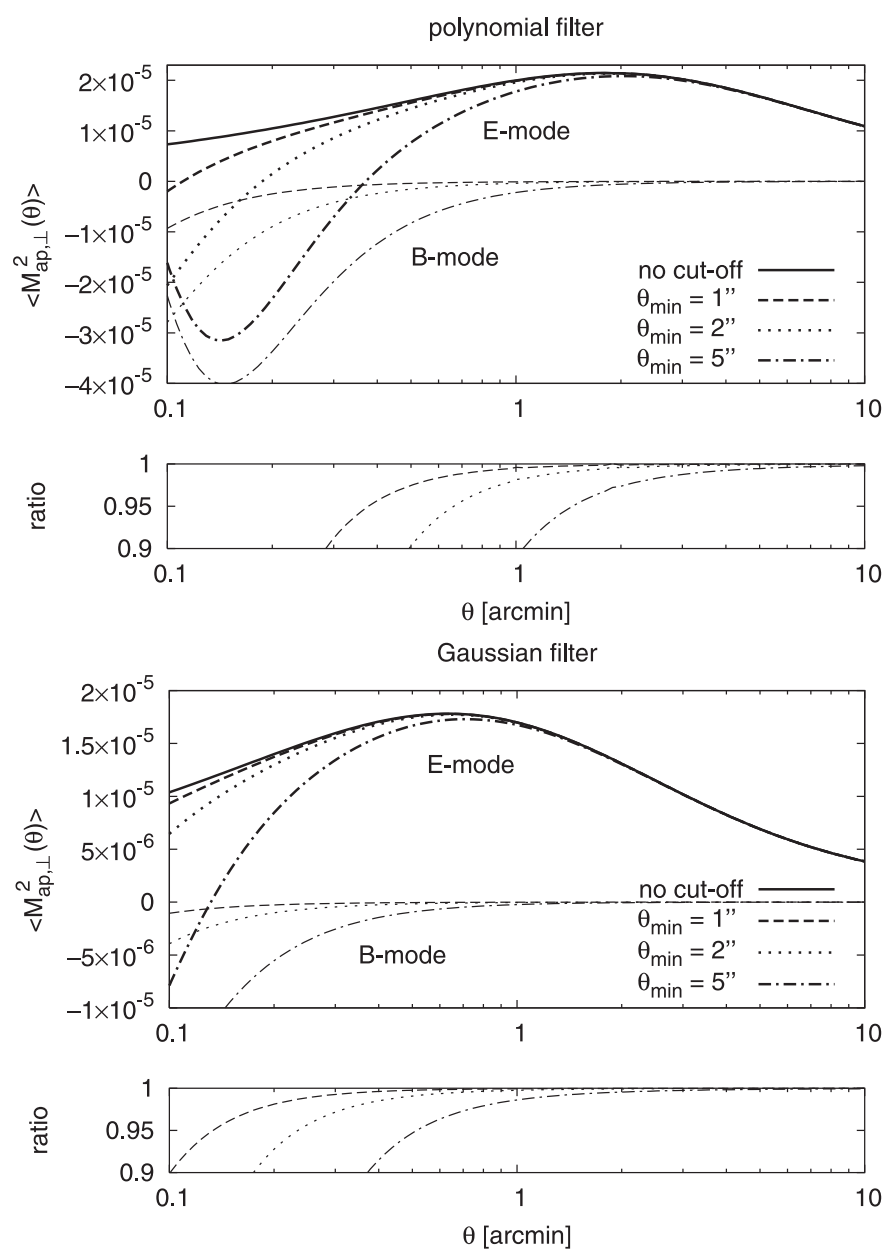

Fig. 1. The E-mode $\left\langle M_{\mathrm{ap}}^{2}(\theta)\right\rangle$ and the leakage from the E-mode $\left\langle M_{\text {ap }}^{2}\left(\theta, \theta_{\min }\right)\right\rangle$ into the B-mode $\left\langle M_{\perp}^{2}\left(\theta, \theta_{\min }\right)\right\rangle$ due to the small-scale cutoff $\theta_{\min }$. The ratio $\left\langle M_{\mathrm{ap}}^{2}\left(\theta, \theta_{\min }\right)\right\rangle /\left\langle M_{\mathrm{ap}}^{2}(\theta)\right\rangle$ is plotted in the small panels.

the following way,

$\xi_{\mathrm{E} \pm}(\theta)=\frac{1}{2}\left[\xi_{+}(\theta)+\xi_{-}(\theta)+\xi_{\mathrm{U}, \mathrm{L}}(\theta)\right] ;$
$\xi_{\mathrm{B} \pm}(\theta)=\frac{1}{2}\left[\xi_{+}(\theta)-\xi_{-}(\theta) \mp \xi_{\mathrm{U}, \mathrm{L}}(\theta)\right]$,

where the integral functions $\xi_{\mathrm{U}}$ and $\xi_{\mathrm{L}}$ correspond to the "+".and "-"-cases, respectively, and are given by

$$
\begin{aligned}
& \xi_{\mathrm{U}}(\theta)=\int_{\theta}^{\infty} \frac{\mathrm{d} \vartheta}{\vartheta} \xi_{-}(\vartheta)\left(4-12 \frac{\theta^{2}}{\vartheta^{2}}\right) \\
& \xi_{\mathrm{L}}(\theta)=\int_{0}^{\theta} \frac{\mathrm{d} \vartheta \vartheta}{\theta^{2}} \xi_{+}(\vartheta)\left(4-12 \frac{\vartheta^{2}}{\theta^{2}}\right) .
\end{aligned}
$$

Thus, in order to separate the E- from the B-modes one needs to know either $\xi_{-}$up to very large or $\xi_{+}$down to very small angular scales. By an unfortunate conspiracy (or maybe not) $\xi_{-}$ falls off rather slowly towards large scales, and $\xi_{+}$is dominant for $\vartheta \rightarrow 0$. Since a small-scale cutoff $\theta_{\min }$ due to close projected galaxy pairs, as well as a maximum scale $\theta_{\max }$ due to the finite observed field are unavoidable, an exact E-/B-mode separation using the above-defined correlation functions is not possible.

Similar to the case of the aperture-mass dispersion (Sect. 2), we define the functions $\xi_{\mathrm{E}, \mathrm{B}+}\left(\theta, \theta_{\max }\right)$ and $\xi_{\mathrm{E}, \mathrm{B}+}\left(\theta, \theta_{\min }\right)$. In the first case, the infinite integral for $\xi_{\mathrm{U}}(10)$ is truncated at $\theta_{\max }$;

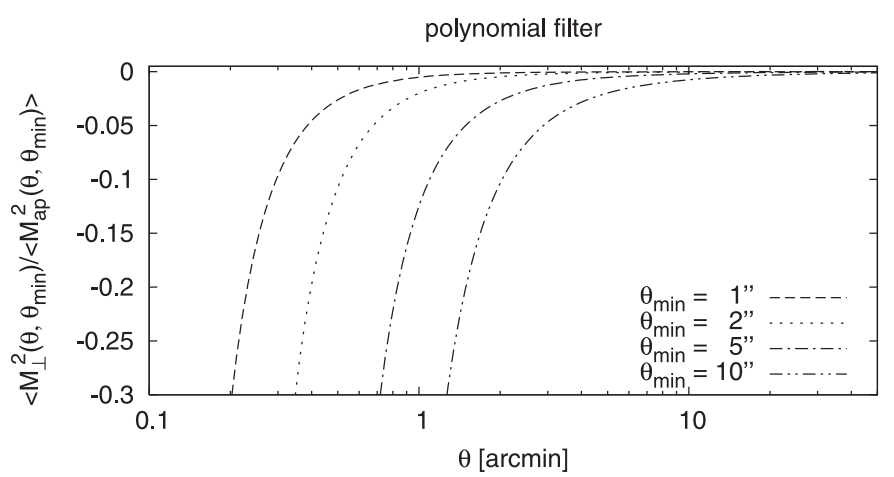

Fig. 2. The B- to E-mode ratio for various small-scale cutoffs $\theta_{\min }$.

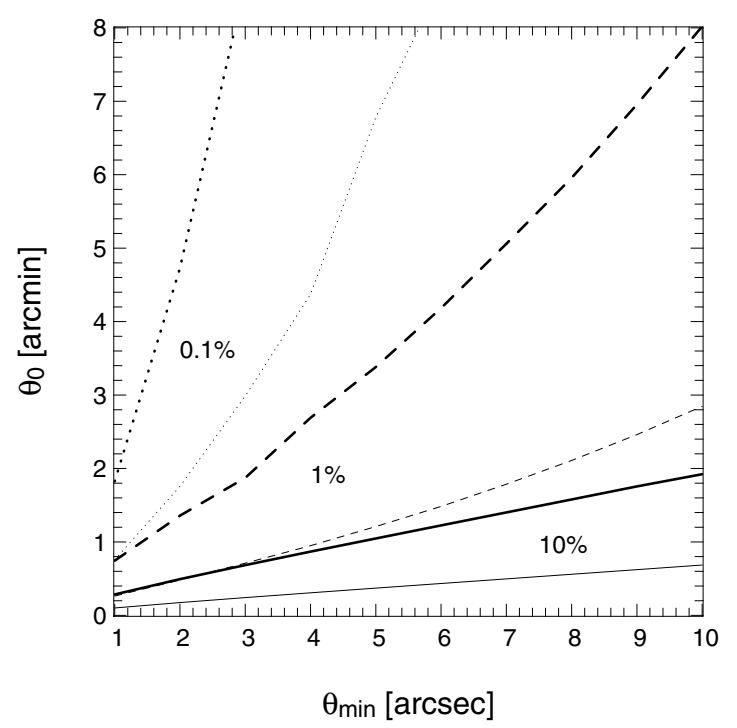

Fig. 3. On scales $\theta$ larger than $\theta_{0}$, the ratio $\left\langle M_{\text {ap }}^{2}\left(\theta, \theta_{\min }\right)\right\rangle /\left\langle M_{\text {ap }}^{2}(\theta)\right\rangle$ is lower than $1-p$, with lines corresponding to $p=10 \%, 1 \%$, and $0.1 \%$ plotted as a function of the cutoff scale $\theta_{\min }$. Thick curves correspond to the polynomial filter (4), thin lines to the Gaussian functions (5).

in the second case, the lower integration limit for $\xi_{\mathrm{L}}$ is replaced by $\theta_{\min }$.

In Fig. 4, the mixing of the E- and B-mode correlation functions is displayed. The large-scale cutoff leads to an underestimation of the true E-mode on all scales, even down to arc seconds. At the same time, a spurious B-mode $\xi_{\mathrm{B}+}$ is introduced. Even for a maximum angular separation of 1.5 degrees, the E-mode is off by more than one percent on all relevant scales. The "minus"-E-mode $\xi_{\mathrm{E}-}$ suffers badly from the close pair cutoff on scales as large as several arc minutes.

Figures 5 and 6 show the deviation of the corrupted E-mode $\xi_{\mathrm{E}+}\left(\theta, \theta_{\max }\right)$ and $\xi_{\mathrm{E}-}\left(\theta, \theta_{\min }\right)$ from the true E-mode $\xi_{\mathrm{E}+}(\theta)$ and $\xi_{\mathrm{E}-}(\theta)$, respectively.

\section{Dependence on cosmology and source galaxy redshift}

The amplitude and the shape of the shear statistics considered here depend, of course, on the underlying cosmology and the resdhift distribution of source galaxies. However, the ratio of the observed E-mode in the presence of the small-scale cutoff $\theta_{\min }$ to the true E-mode $\left(\left\langle M_{\mathrm{ap}}^{2}\right\rangle, \xi_{\mathrm{E}, \mathrm{B}-}\right)$ is very insentive to the convergence power spectrum. In the case of $\xi_{\mathrm{E}, \mathrm{B}+}$, the difference between the E-mode corresponding to a maximal scale $\theta_{\max }$ and 

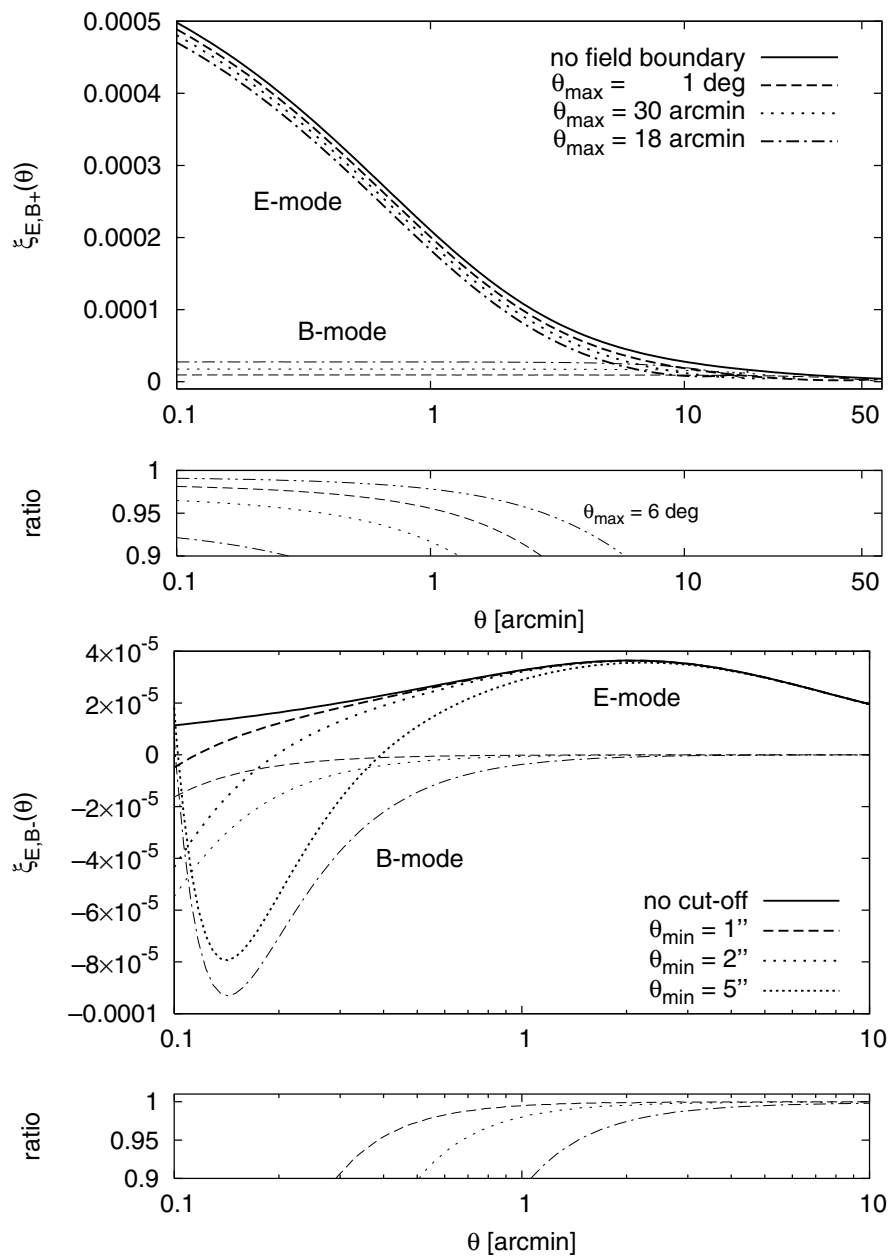

Fig. 4. The E-/B-mode correlation functions. Top panels: mixing between $\xi_{\mathrm{E}+}$ and $\xi_{\mathrm{B}+}$ due to a finite field. The curves correspond to an infinite field and to fields with maximal scales of $\theta_{\max }=1 \mathrm{deg}, 30$, and 18 arcmin as indicated in the panel. (The E- and B-mode for $\theta_{\max }=6 \mathrm{deg}$ is not shown in the upper panel.) Bottom panels: mixing of $\xi_{\mathrm{E}, \mathrm{B}-}$ due to a small-scale cutoff $\theta_{\min }$. In the small panels, the ratio between the corrupted and the true E-mode is shown.

the true E-mode depends only little on the underlying model. However, the amplitude of $\xi_{\mathrm{E}+}$ depends quite strongly on the amplitude of the power spectrum, which is governed in particular by $\Omega_{\mathrm{m}}, \sigma_{8}$, and the source redshift distribution. As a consequence, the ratio of the observed to true E-mode depends on those parameters. For a deeper survey, this ratio will be lower than for a more shallow one. For all plots in this paper, if not indicated otherwise, a mean redshift of $\bar{z}=1.0$ is assumed. The cosmology is $\Lambda$ CDM with $\Omega_{\mathrm{m}}=0.3$ and $\sigma_{8}=0.85$.

\section{Summary and conclusions}

We have quantified the mixing between the E- and the B-modes which arises due to the lack of information about the shear correlation on both very small and very large scales. The former limit $\theta_{\min }$ is due to close projected pairs, the shape of which cannot be determined reliably. The latter cutoff $\theta_{\max }$ is related to the finite size of the observed galaxy fields. Apart from that, a fundamental limit exists since only a $4 \pi$-survey can in principle yield an unambiguous E- and B-mode decomposition which, however, is unrealistic due to the occlusion of parts of the sky by the Milky Way (Bunn 2003). We used the aperture-mass dispersion $\left\langle M_{\mathrm{ap}, \perp}^{2}\right\rangle$

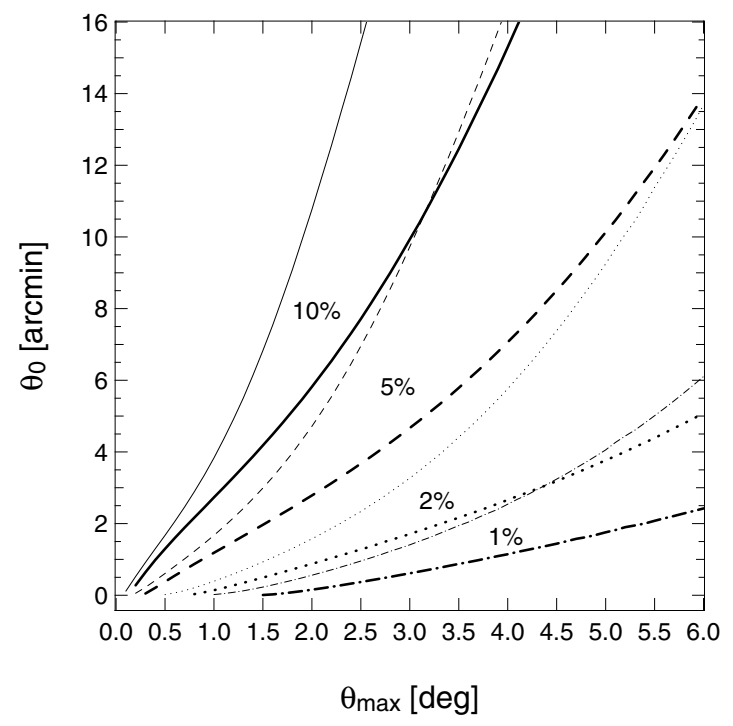

Fig. 5. On scales $\theta$ smaller than $\theta_{0}$, the ratio $\xi_{\mathrm{E}+}\left(\theta, \theta_{\max }\right) / \xi_{\mathrm{E}+}(\theta)$ is lower than $1-p$, where lines corresponding to $p=10,5,2$, and $1 \%$ are plotted as a function of the maximum separation $\theta_{\max }$. Thick curves correspond to a mean source redshift of $\bar{z}=1$, thin lines to $\bar{z}=1.5$.

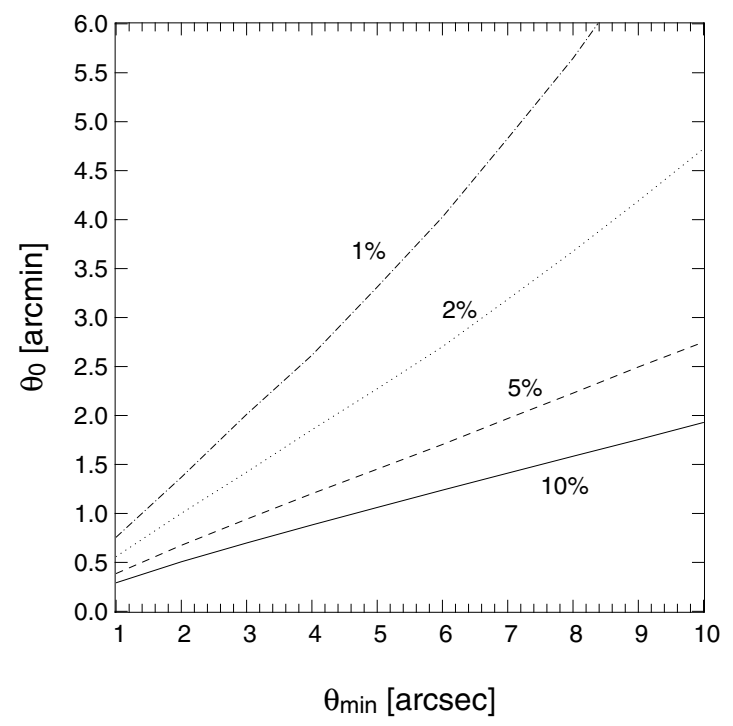

Fig. 6. On scales $\theta$ larger than $\theta_{0}$ the ratio $\xi_{\mathrm{E}-}\left(\theta, \theta_{\max }\right) / \xi_{\mathrm{E}-}(\theta)$ is smaller than $1-p$, where lines corresponding to $p=10,5,2$ and $1 \%$ are plotted as a function of $\theta_{\min }$.

and the shear-correlation functions $\xi_{\mathrm{E}, \mathrm{B} \pm}$ as measures of the Eand B-modes, respectively.

Even if the cutoff due to close galaxy pairs is as small as 5", which is feasible for ground-based observations, the E-mode is underestimated by $10 \%$ (1\%) on scales below 1' (3.4), using $\left\langle M_{\mathrm{ap}}^{2}\right\rangle$ with the polynomial filter function. Moreover, a negative B-mode signal appears. Since even with space-based measurements, a cutoff of about $\theta_{\min }=1^{\prime \prime}$ exists due to blending galaxy images, it is fundamentally not possible to know whether there is a B-mode present on scales smaller than one or two arc minutes with very high (sub-percent) precision. This cutoff does not introduce a B-mode into the $2 \mathrm{PCF}$, however, it prevents the clear detection of any B-mode still present in the data that may corrupt the $2 \mathrm{PCF}$ on much larger scales than $\theta_{\min }$. 
The E-/B-mode correlation function $\xi_{\mathrm{E}, \mathrm{B}-}$ behaves in a similar way than $\left\langle M_{\mathrm{ap}}^{2}\right\rangle$. The "+"-version, $\xi_{\mathrm{E}, \mathrm{B}+}$, on the other hand is affected by a large-scale cutoff $\theta_{\max }$ that causes a leakage from the E- to the B-mode on all angular scales. For fields smaller than $1.5 \mathrm{deg}$, the precision of the E-mode correlation function is never better than $1 \%$.

In order to obtain knowledge about the B-mode in the data, one has to combine $\xi_{\mathrm{E}, \mathrm{B}+}$ and $\xi_{\mathrm{E}, \mathrm{B}-\text {. For ground-based data }}$ $\left(\theta_{\min }=5^{\prime \prime}\right), \xi_{\mathrm{E}, \mathrm{B}-}$ allows one to constrain the B-mode at the percent level on scales larger than 3.3. To get this precision on larger scales using $\xi_{\mathrm{E}, \mathrm{B}+}$, the measurement of the shear correlation up to about $\theta_{\max }=7$ degrees is necessary. From space-based observations with $\theta_{\min }=1^{\prime \prime}, \theta_{\max }=3.4 \mathrm{deg}\left(\theta_{\max }=2.4 \mathrm{deg}\right)$ is needed for a mean redshift of $\bar{z}=1.0(\bar{z}=1.5)$, respectively.

Up to now, various strategies have been employed to estimate the E- and B-mode from shear data. Massey et al. (2005) calculate the E-/B-mode correlation functions by extrapolating $\xi_{-}$beyond measured scales, using a theoretical model for $P_{\kappa}$. However, this method explicitely makes the assumption that no B-mode is present on larger scales than probed by the survey. Choosing a wrong model can change the amplitude of $\xi_{\mathrm{E}}$ on the measured angular range, which might bias the cosmological interpretation of the shear correlation. In particular, $\Omega_{\mathrm{m}}$ and $\sigma_{8}$ might be biased, which, although within the systematic errors for current measurements (see Fig. 10 of Heymans et al. 2005), will be problematic for future surveys.

Recently, van Waerbeke et al. (2005) use the aperture-mass dispersion to calibrate the E-mode shear-correlation function. Since their measured B-mode is consistent with zero on large scales $\left(10^{\prime}-50^{\prime}\right)$, they shift $\xi_{\mathrm{E}, \mathrm{B}}$ such that the B-mode vanishes on corresponding angular scales $\left(2^{\prime}-10^{\prime}\right)$. It has to be noted that a B-mode on very large scales does not have any influence on $\left\langle M_{\mathrm{ap}}^{2}\right\rangle$ on smaller scales, but it will corrupt the correlation function. Moreover, there is no clear correspondence between the angular scales probed by the correlation function and the aperturemass dispersion.

Alternatively, the aperture-mass statistics can be measured and fitted with a cosmological model (e.g. Jarvis et al. 2003). In that case, either scales below $\theta_{0}$ have to be omitted from the analysis, or the theoretical prediction of $\left\langle M_{\mathrm{ap}}^{2}\right\rangle$ has to be obtained using Eq. (6) with the cutoff $\theta_{\min }$ specified by the observations. Note, however, that in this case the B-mode signal should also be fitted with the predicted, non-zero $\left\langle M_{\perp}\left(\theta, \theta_{\min }\right)\right\rangle$ if no lensing information is to be lost.
At present, the prediction on the shear signal at angular separations on arc-minute scales and below is very difficult and inaccurate. These small scales should not be disregarded since the shear signal on these scales is very high and contains unique information about the non-linear and non-Gaussian features of the large-scale structure. In the future, when our non-linear models of structure formation improve and baryonic effects are taken into account, shear measurements on small scales will be of great use. Since the shear signal on small scales is particular clear separation between the E- and B-mode on these scales is manly susceptible to contamination by intrinsic galaxy alignment, adatory. Only then can one make certain that the measurement is free of systematic errors and has the necessary quality to be used for precision cosmology.

Acknowledgements. We thank Marco Hetterscheidt, Tim Schrabback-Krahe, and Richard Massey for fruitful discussions, and the anonymous referee for useful comments.

\section{References}

Bunn, E. F. 2003, New Astron. Rev., 47, 987

Crittenden, R. G., Natarajan, P., Pen, U.-L., \& Theuns, T. 2001, ApJ, 559, 552 Crittenden, R. G., Natarajan, P., Pen, U.-L., \& Theuns, T. 2002, ApJ, 568, 20

Heymans, C., \& Heavens, A. 2003, A\&A, 339, 711

Heymans, C., Brown, M. L., Barden, M., et al. 2005, MNRAS, 361, 160

Heymans, C., van Waerbeke, L., et al. 2006a, accepted by MNRAS

[arXiv: astro-ph/0506112]

Heymans, C., White, M., Heavens, A., Vale, C., \& van Waerbeke, L. 2006b, MNRAS, submitted [arXiv: astro-ph/0604001]

Hirata, C. M., \& Seljak, U. 2004, Phys. Rev. D, 70, 063526

Hirata, C. M., Mandelbaum, R., Seljak, U., et al. 2004, MNRAS, 353, 529

Hoekstra, H., Mellier, Y., van Waerbeke, L., et al. 2006, A\&A, 647, 116

Jarvis, M., Bernstein, G., Fischer, P., \& Smith, D. 2003, AJ, 125, 1014

Jarvis, M., Jain, B., Bernstein, G., \& Dolney, D. 2006, ApJ, 644, 71

Jing, Y. P. 2002, MNRAS, 335, L89

Kaiser, N., Squires, G., Fahlman, G., \& Woods, D. 1994, in Clusters of galaxies, Proceedings of the XIVth Moriond Astrophysics Meeting, Méribel, France, 269 [arXiv: astro-ph/9407004]

King, L. J., \& Schneider, P. 2002, A\&A, 396, 411

King, L. J., \& Schneider, P. 2003, A\&A, 398, 23

King, L. J. 2005, A\&A, 441, 47

Massey, R., Refregier, A., Bacon, D. J., Ellis, R., \& Brown, M. L. 2005, MNRAS, 359, 1277

Rhodes, J., Refregier, A., Collins, N. R., et al. 2004, ApJ, 605, 29

Schneider, P. 1996, MNRAS, 283, 837

Schneider, P., van Waerbeke, L., Jain, B., \& Kruse, G. 1998, MNRAS, 296, 873

Schneider, P., van Waerbeke, L., \& Mellier, Y. 2002, A\&A, 389, 729

Semboloni, E., Mellier, Y., van Waerbeke, L., et al. 2006, A\&A, 452, 51

van Waerbeke, L., Mellier, Y., \& Hoekstra, H. 2005, A\&A, 429, 75 